\title{
ENFOQUE TÉCNICO EN LA ADMINISTRACIÓN DE RIESGOS EMPRESARIALES (2014)
}

\author{
FOCUS ON TECHNICAL ENTERPRISE RISK MANAGEMENT (2014)
}

Félix Armando Rivera León*

felix_arl@hotmail.com

[RECEPCIÓN: AGOSTO 2015 / CONFORMIDAD: NOVIEMBRE 2015]

\section{RESUMEN}

Actualmente los riesgos en los niveles empresariales, las oportunidades de inversión y las oportunidades personales toman un grado importante dentro de la gestión administrativa en pro de los objetivos de la misma. Nuestro estudio trata de resolver cómo se construye una estrategia empresarial, teniendo presente la existencia de un conjunto de prácticas que aunque no garantizan que al final se tenga un buen resultado, si sirven como marco de referencia para organizar el proceso y lograr un enfoque técnico realizable.

Palabras clave: Riesgos empresariales, enfoque.

\section{ABSTRACT}

Currently the risk levels in business, investment opportunities and personal opportunities; today, they are taking a significant degree within the administration towards the same goals. Our study is figuring out how to build a business strategy, bearing in mind the existence of a set of practices but do not guarantee that the end result will be good, if it serves as a framework to organize the process and make a technical approach feasible.

Keywords: Business risk, approach.

* Doctor en Ciencias Contables y Empresariales por la Universidad Nacional Mayor de San Marcos (UNMSM), Magíster en Administración con mención en Gestión Empresarial (UNMSM), Contador Público Colegiado con 37 años de experiencia profesional, profesor principal de la Facultad de Ciencias Administrativas de la UNMSM. Ha desempeñado diversos cargos administrativos en la facultad y en diferentes entidades del sector público y privado. 


\section{INTRODUCCIÓN}

Los modelos orientados a la gestión financiera, de calidad, ambiental, seguridad de información, responsabilidad social empresarial, continuidad del negocio, entre otros, son herramientas que permiten la articulación de los sistemas que representan una estrategia institucional.

Lograr la interacción es uno de los retos más importantes para la alta dirección y su equipo de trabajo, pues con ello se busca efectuar una planeación estratégica integrada, mediante el empleo incluso de requisitos normativos, donde los procedimientos y los trabajadores deberán estar en sintonía para un eficiente, eficaz y efectivo logro de resultados. Lamentablemente, una de las debilidades que se ha podido evidenciar durante mi experiencia profesional, es que a veces se planea el desarrollo de objetivos, donde el resultado final es otro totalmente diferente al que inicialmente se definió, invirtiendo cuantiosos recursos e incumpliendo los presupuestos inicialmente previstos.

Actualmente los riesgos en los niveles empresariales, las oportunidades de inversión y oportunidades personales toman un grado importante dentro de la gestión administrativa en pro de los objetivos de la misma, dado que existe la creciente necesidad de conocer y manejar los niveles de incertidumbre a los que está expuesta la empresa durante la ejecución de las estrategias y el cumplimiento de los objetivos y metas, debido especialmente al grado de globalización en el que nos insertamos, lo que ha ampliado el espectro de oportunidades así como los riesgos a los que se enfrentan a diario.

Nuestro estudio trata de resolver cómo se construye una estrategia empresarial, teniendo presente la existencia de un conjunto de prácticas que aunque no garantizan que al final se tenga un buen resultado, sí sirven como marco de referencia para organizar el proceso y lograr un enfoque técnico realizable.

La meta que brinda el estudio permite aplicar conceptos relacionados con la administración de riesgos a situaciones de gestión empresarial, estableciendo puentes entre lo académico y la práctica.

La metodología presenta conceptos aplicados en las aulas universitarias y en empresas, y de soporte aplicamos un modelo que correlacione los factores: producto de calidad, satisfacción del cliente, ubicación estratégica en el mercado, logro de eficiencia operacional, posicionamiento de la marca, así como responsabilidad del entorno.

El objetivo general fue lograr un enfoque técnico que permita orientar el logro de estrategias del negocio, con el objetivo de realizar decisiones eficientes dentro del marco de las metas y objetivos de la misma. Específicamente es realizar un manejo apropiado de los recursos empresariales que permitan estrategias de logro orientando la alineación de las mismas, dentro de un enfoque que los relacione hacia metas y objetivos esperados.

En cuanto a las metodologías y técnicas de investigación utilizadas, el trabajo se enmarca dentro del conocimiento previo que presupone la investigación científica, en concordancia con Mario Bunge (1980) en la que constata la validez, existencia y cognoscibilidad del mundo externo. Nuestro estudio está dentro de la esfera técnica y moral al enfocar la problemática así como netamente objetivo realista. Se establece una metodología que permite procedimientos pertinentes dirigidos por reglas que logran conocimiento objetivo que se puedan justificar a manera teórica y empírica.

- El enfoque de la investigación es de tipo abductivo creativo, porque parte de los hechos explicados son de tipo inductivo, que permiten esclarecer una realidad (marco teórico).

- La estrategia de investigación se encuadra en la experiencia laborar del autor y las teorías que fundamentan la especialidad de auditoría.

- Los métodos de colección de datos fueron de características primario/secundario; entrevistas; observaciones.

- El análisis de datos fue de tipo descriptivo y cualitativo.

- El trasfondo filosófico se considera un contexto de demarcación ontológico, gnoseológico así como racional, conceptual y lógico; teórico en los campos de las ciencias y de la técnica, las que constituyen acciones metodológicas.

- En cuanto al aspecto formal es tipo lógico pero consistente.

- El cuerpo del conocimiento está dentro de los alcances de las ciencias sociales, ahí también se encuentran las ciencias administrativas, como las ciencias contables. 
- La muestra se tomó teniendo en cuenta a las empresas consideradas medianas y grandes en Lima Metropolitana (aproximadamente 200); encuestando un funcionario de cada una, en niveles de la alta dirección. El estudio implica validar el instrumento; es por esta razón que se puede realizar un cálculo del tamaño de la muestra para encontrar el número de individuos que participarán en las encuestas preformuladas. La opción será un muestreo no probalístico de tipo discrecional (focus group).

- Se calculó la fiabilidad de los datos mediante el Alfha de Cronbach.

- La validación del instrumento de medición fue mediante la utilización del paquete computarizado SPSS 17 (Statistical Package Social Science).

\section{BASES TEÓRICAS}

Las bases teóricas en las que se sostiene el presente estudio se fundamentan en las siguientes conceptualizaciones:

En el mundo empresarial, nuestros empresarios poseen una cultura de gestión administrativa cuyo resultado es la toma de decisiones, diversas y heterogéneas magnitudes económicas para con sus empresas bajo responsabilidad.

El presente estudio está orientado a establecer en una u otra forma un enfoque metodológico en el que se obtengan, en lo posible, resultados eficientes como consecuencia de las tomas de decisiones en el organismo de la empresa. Para ello requerimos partir del concepto muy propio de la expresión: administrar, por lo que se ideará y desarrollará un constructo conceptual.

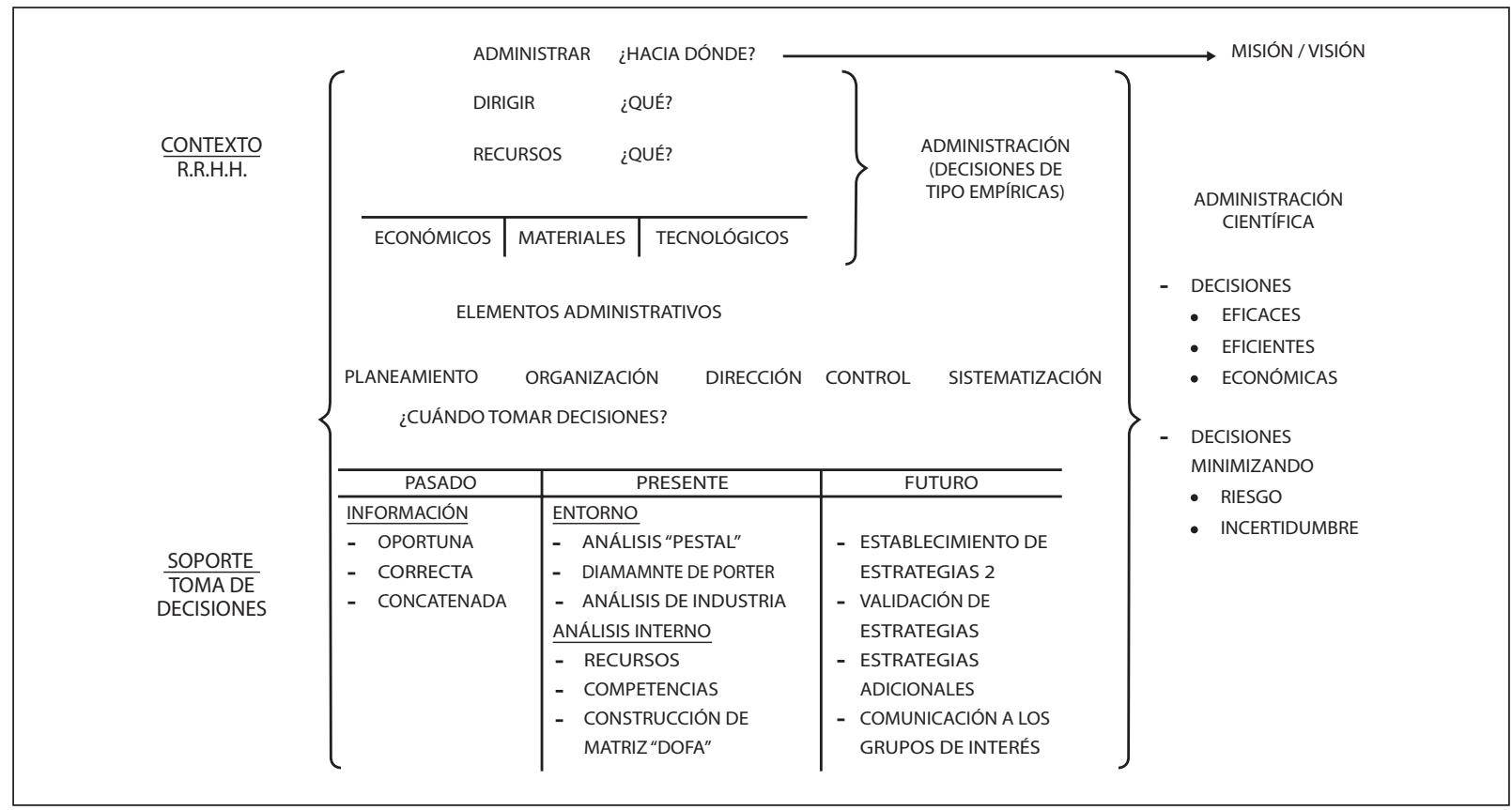

Fig. №1. Gestión administrativa

\section{ANÁLISIS DEL ENTORNO EXTERNO}

En el análisis del entorno externo de la empresa debemos considerar el empleo de tres referencias teóricas:

\section{Análisis PESTEL}

Organiza los aspectos más relevantes del entorno y la forma cómo pueden afectar la viabilidad futura; reconoce cinco factores, necesarios a tomar en cuenta cuando se discute un proyecto:

\section{- Político}

- Económico

- Sociocultural

- Tecnológico

- Legal

\section{Diamante de Porter}

Permite reconocer las condiciones existentes en una región o en un país, respecto al segmento de mercado, facilitando o dificultado la operación que se desea realizar. 
- Condiciones de facturas (recursos, capital humano, infraestructura, seguridad y ubicación geográfica).

- Industrias relacionadas y de apoyo.

- Condición de la demanda: (precios, nivel de exigencia de los consumidores, variación de gastos y necesidades).

- Estrategia, estructura y rivalidad empresarial.

- Gobierno (estabilidad fiscal, transparencia de instituciones, regulación, estabilidad jurídica, respeto a los acuerdos y contratos).

\section{Análisis de la industria}

En el contexto del estudio es necesario analizar con el fin de determinar el grado de rivalidad y los factores críticos de éxito necesarios para sobrevivir y ganar participación de mercado.
Las fuerzas de Porter (1991) son las siguientes:

- Poder de los proveedores.

- Barreras de entrada.

- Temor de sustitutos.

- Poder de compradores.

- Poder de los clientes.

- Poder de los colaboradores.

\section{ANÁLISIS DEL ENTORNO INTERNO DE LA EMPRESA}

El análisis del entorno interno de la empresa constituye el manejo de recursos y competencias, identificando debilidades o riesgos internos en el contexto de la industria previamente establecida, analizado bajo enfoques de causa-efecto y funciones principales de gestión y soporte.

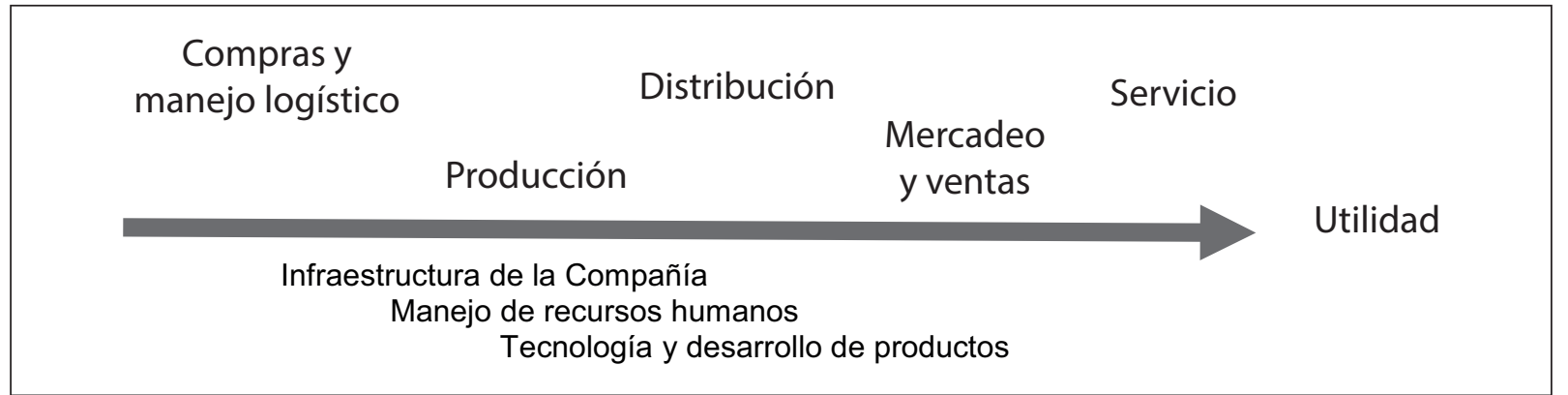

Fig. $\mathrm{N}^{\circ} 2$. Análisis del entorno interno de la empresa

Dentro de las áreas a analizar encontramos:

- Activos físicos: Plantas, equipos y propiedades.

- Activos financieros: Efectivo y posibilidades de apalancamiento.

- Recursos humanos: Conocimientos, habilidades personales, lealtad.

- Activos intangibles: Marcas, tecnología, reputación.

- Activos organizacionales: Cultura, sinergias y habilidades de trabajo en equipo.

La matriz FODA es parte de la investigación requerida y de las decisiones que permitan consignar en la misma debilidades, oportunidad, fortalezas y amenazas; la cual es de suma utilidad para la identificación de los riesgos externos e internos que afronta la empresa que se desea proyectar. Será de vital importancia para establecer las estrategias que se deberán trabajar a fin de sortear con éxito los ataques de la competencia, aprovechar las oportunidades que existan, establecer alianzas, etc.

\section{ESTRATEGIAS GENÉRICAS}

Otro de los puntos importante son las estrategias genéricas, las que deben establecer las ventajas competitivas que permitan a la empresa sobrevivir en medio de la competencia del sector.

Existen dos tipos de estrategias genéricas, según Michael Porter:

- Bajos costos.

- Venta de productos diferenciados por los que el consumidor esté dispuesto a pagar.

Establecer estas estrategias genéricas obedece a la realidad del mercado, que es lograr entregar al cliente con los mismos beneficios pero a menor costo, u ofrecer mejores servicios que sus competidores no pueden ofertar. Para esto es necesario tomar en cuenta: 


\section{- Definición de ventajas competitivas}

De acuerdo con la matriz FODA, la organización puede definir ventajas competitivas y estrategias de acción para vencer a la competencia.

- Estrategias F-O

- Estrategias D-O

- Estrategias F-A

- Estrategias D - A

\section{- Factores claves del éxito}

Podríamos considerar lo siguiente, como ejemplo:

- Producto de alta calidad.

- Lo primero es el cliente y la satisfacción de sus necesidades.

- Estratégica ubicación en el mercado.

- Eficiencia operacional.
- Posición de la marca.

- Responsabilidad con el entorno.

\section{- Establecimiento de objetivos y metas}

- Definida la estrategia empresarial, se deberá establecer medidas que permitan el monitoreo y control de las acciones conducentes a su cumplimiento. El fijar indicadores que permitan controlar el alcance de las metas, es importante.

- El alineamiento de recursos en forma estratégica, debe ser claro, redunda en beneficio de los accionistas, directivos y partes interesadas, en la medida que todos saben a dónde quiere ir la empresa y que acciones se deben emprender para lograrlo.

- Es lógico que dentro del planteamiento del estudio interno orgánico empresarial se establezca claramente:

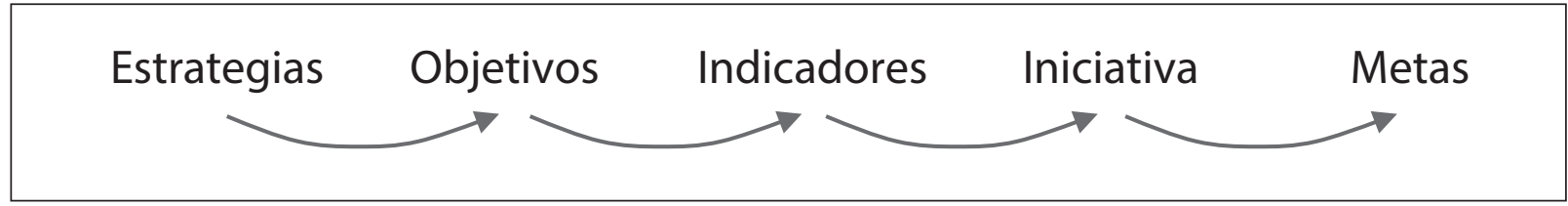

\section{- Declaración de la Misión}

Es tener clara la razón de ser la empresa. Una idea fundamental es que esté por encima de la estrategia, los objetivos y las metas, que se mantenga firme a pesar de los cambios que pueda tener en el futuro, por las circunstancias, variables del mercado y la competencia.

\section{- Declaración de la Visión}

Permite establecer los principios fundamentales y permanentes de la organización.

Las empresas que perduran en el tiempo son las que poseen una ideología fundamental que da lugar a mantener unido y motivado al personal durante su crecimiento y desarrollo, a pesar de los cambios futuros en las estrategias. Se conoce como meta grande y audaz, son valores que direccionan el actuar de la empresa y que permite obtener su propia identidad.

- En cuanto al riesgo, elemento básico en la toma de decisiones, se debe conceptuar, visto desde el argumento empresarial: El riesgo empresarial es la posibilidad de que los flujos de efectivo de una empresa sean insuficientes para cubrir los gastos de operación. Afecta negativamente el valor por lo que es necesario administrar el riesgo, orientado a maximizar el valor del negocio.

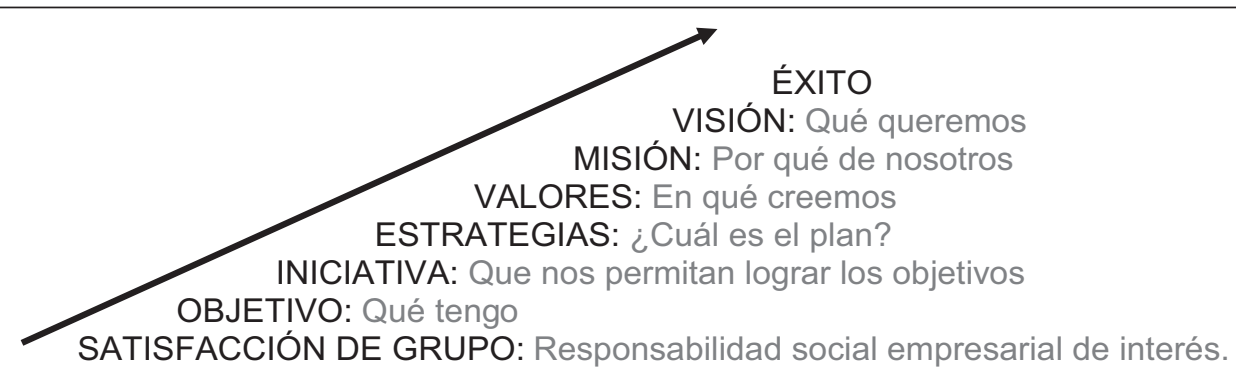


Una administración de riesgos eficiente se traduce en efectos económicos, constituye una herramienta para la toma de decisiones, es consecuente a los objetivos y metas dentro de los lineamientos previstos por la organización en el logro de la visión empresarial.

El estudio pretende y tiene como objetivo general diseñar y aplicar un enfoque orientado a lograr una metodología para la administración de riesgos bajo los requisitos de la norma ISO 9000 y garantizar la fiabilidad de la información económica- financiera.

El aporte teórico consiste en lograr un enfoque metodológico para la administración de riesgos empresariales que correlacione marcos teóricos como control interno, gestión de calidad, análisis débiles y críticos de control. Tomando en cuenta el análisis y evaluación de los riesgos:

- Administración de riesgos.

- Proceso de la administración de riesgos.

- Técnicas de procedimientos para administrar riesgos.

- Evaluación de riesgos.

\section{CONSIDERACIONES PARA LA PRÁCTICA DE EVALUACIÓN EN BASE A RIESGOS}

- Objetivos de la empresa, organización, estructura, políticas, normas, legislación aplicable, etc.

- Operaciones y procesos que se llevan a cabo para la consecución de los fines.

- Identificación de la importancia relativa de las diversas operaciones así como de los procedimientos y grado de automatización para llevarlas a cabo.

La unidad de análisis está constituida por empresas e instituciones del medio en Lima Metropolitana, las mismas que poseen auditorias previamente realizadas. Sin embargo, como no se trata de un estudio que pretenda generalizaciones para todo el universo; sino más bien, para el logro de las encuestas, nos bastó trabajar con una población seleccionada y estratificada, que permitió condiciones de desarrollo de nuestros resultados.

Se deberá entender que los resultados a que llegó nuestra investigación concuerdan en general con los puntos de vista expuestos usualmente en la literatura. Representaron nuestra conceptua- lización del problema, en base a la bibliografía existente, como en los cambios de ideas con profesionales de la alta dirección empresarial, y en nuestra propia experiencia.

\section{Del análisis}

Un eficiente trabajo de dirección empresarial puede ser garantizado en la medida que la organización conozca la operatividad y se apoye en una metodología que norme la gestión.

Los niveles de productividad de las instituciones en general podrán mejorar en la medida que las carencias y deficiencias sean detectadas a tiempo a través de un planeamiento oportuno.

La gestión administrativa en las empresas se basa en los objetivos, en los recursos y en el sistema de información implementado.

Ello dependerá de la apropiada formación profesional de los directivos a cargo; a quienes se entrevistarán mediante los instrumentos pertinentes; por consiguiente, es el sujeto en estudio en la presente investigación.

\section{MÉTODO DE LA INVESTIGACIÓN}

El método de la investigación es Inductivo. La realización de la observación se realizó mediante encuestas, cuestionarios previamente confeccionados, con el objeto de que dichos elementos de juicio cumplan una función de enlace entre los objetivos de la investigación y la realidad de la población observada, por ello se realizó a través de preguntas concretas sobre dicha realidad, y por otra parte debe ser capaz de suscitar en los encuestados respuestas sinceras y claras a cada pregunta, con el objeto de que puedan ser clasificadas y analizadas.

\section{Selección de la muestra}

El universo poblacional está constituido por cinco empresas de servicio público: Luz del Sur S.A.A., EDELNOR S.A.A., EDEGEL S.A.A. SEDAPAL S.A.A., Telefónica del Perú S.A.A.; cuatro empresas administradoras de fondos de pensiones: Integra, Prima, Profuturo, Habitat, constituidas en Lima Metropolitana, consideradas en el estudio por su materialidad económica y funcional en la región.

De ellas se tomó como sujetos en investigación a directivos, profesionales autorizados en el marco direccional del planeamiento en sus respectivas empresas; así como de la realización, desarrollo y seguimiento de las mismas. 
Así mismo, se consideran a las 33 universidades públicas y privadas de Lima Metropolitana, lo que significa en su conjunto el alcance del estudio.

\section{Tamaño de la muestra}

Para Torres (1999: 187-188) "[...]la muestra puede ser determinada por sectores (racimos) geográficos, sociales, educativos, económicos, etc., siempre y cuando los elementos del universo tengan características comunes".

La muestra fue la misma población establecida como alcance en el estudio, por lo que no requeriremos establecer cantidad alguna mediante fórmula. Dado que se investiga a todo el conjunto de sujetos (población), será de tipo censal; ya que la población es pequeña, por lo que no es necesario determinar la muestra.

\section{Construcción del instrumento de medición}

Se determina la confección de los cuestionarios según la naturaleza de trabajo de cada personaje implicado, utilizado como medida de valor la tabla Likert ( 5 a 1 en forma decreciente).

Una vez realizada en su totalidad la encuesta, se procedió al análisis de los datos, con el objetivo de determinar las dimensiones e ítems más representativos y, de esta forma, confirmar cuáles son los factores de desarrollo y calidad en la investigación.

Uno de los coeficientes más comunes para calcular la fiabilidad es el Alpha de Cronbach (Cronbach, 1951: 297-334) que se orienta hacia la consistencia interna de una prueba y refleja el grado en que convocarían las preguntas que constituyen la escala. Para valores inferiores a 0,6 se considera una baja fiabilidad. Entre 0.6 y 0.8 es aceptable. Por encima de 0,8 es excelente, indicará una alta homogeneidad y equivalencia de respuesta a todos los ítems a la vez y para todos los encuestados (Díaz, 2003).

Se utilizó como validez temática el software de soporte el SPSS, versión 22, habiéndose utilizado las pruebas estadísticas de correlación Pearson; coeficiente del modelo de regresión múltiple; tablas de frecuencias; F de Fischer (análisis de varianza ANOVA); $t$ de student (coeficiente del modelo).

\section{RESULTADOS Y DISCUSIÓN}

\section{Planteamos aquí nuestras hipótesis}

\section{Hipótesis general}

La efectividad de las estrategias empresariales coadyuvaran hacia la eficiencia si para cada nivel organizacional estratégico, táctico u operativo y de soporte, se alinearán perspectivas en los ámbitos: financieros, cliente, internos, y el mejoramiento continuo, aplicando un enfoque metodológico que correlacione productos de calidad, satisfacción del cliente, ubicación en el mercado, logro de eficiencia operativa.

\section{Primera hipótesis específica}

La rentabilidad económica financiera, el valor de la empresa, su liquidez y nivel de utilidades, así como el control apropiado de costos, coadyuvan en las perspectivas de estrategias eficientes en la empresa.

\section{Segunda hipótesis específica}

Los niveles de venta y de los precios así como, la calidad del producto y su dominio de la marca, considerando la ubicación en el mercado y la satisfacción del cliente promueven la creatividad de estrategias eficientes en la empresa.

\section{Tercera hipótesis específica}

Las estrategias eficientes dependerán en su marco interno de los niveles de calidad de los procesos, del logro de certificación de los mismos, que éstos garanticen la protección del medio ambiente, el logro de reducción en tiempos y movimientos, de la limpieza e higiene existente, del modo del aseguramiento del proveedor, y de los precios justos a los proveedores.

\section{Cuarta hipótesis específica}

El apropiado mejoramiento continuo de los recursos humanos coadyuvará a una eficiente elaboración de estrategias empresariales, tomando en cuenta la capacitación, selección de personal, clima laboral, cultura de servicio y atención al cliente, así como apoyo al cliente.

\section{ANÁLISIS, INTERPRETACIÓN Y DISCUSIÓN DE RESULTADOS}

\section{Confiabilidad del instrumento de medición}

Considerando una muestra piloto, que fue contestada por 30 personas, se obtuvo un co- 
eficiente de confiabilidad de Alfa de Cronbach de 0.739 . Este valor denota la confiabilidad del mismo.

\begin{tabular}{|c|c|}
\hline \multicolumn{2}{|c|}{ Estadísticos de fiabilidad } \\
\hline Alfa de Cronbach & N. ${ }^{\circ}$ de elementos \\
\hline, 739 & 21 \\
\hline
\end{tabular}

Cada una de las preguntas del cuestionario fue respondida mediante escala de Likert con las siguientes puntuaciones:

1. Totalmente en desacuerdo

2. En desacuerdo

3. Ni de acuerdo ni en desacuerdo

4. De acuerdo

5. Totalmente de acuerdo

\section{Análisis descriptivo}

La perspectiva financiera fue medida a través de cuatro ítems, con un puntaje total como el que se muestra en la tabla 1 :

\begin{tabular}{|c|c|c|c|c|}
\hline \multicolumn{5}{|c|}{ Tabla 1. Perspectiva financiera } \\
\hline & & Frecuencia & Porcentaje & Porcentaje acumulado \\
\hline \multirow{11}{*}{ Válidos } & 10 & 3 & 3.9 & 3.9 \\
\hline & 11 & 1 & 1.3 & 5.3 \\
\hline & 12 & 2 & 2.6 & 7.9 \\
\hline & 13 & 13 & 17.1 & 25.0 \\
\hline & 14 & 7 & 9.2 & 34.2 \\
\hline & 15 & 11 & 14.5 & 48.7 \\
\hline & 16 & 11 & 14.5 & 63.2 \\
\hline & 17 & 14 & 18.4 & 81.6 \\
\hline & 18 & 11 & 14.5 & 96.1 \\
\hline & 19 & 3 & 3.9 & 100.0 \\
\hline & Total & 76 & 100.0 & \\
\hline
\end{tabular}

De acuerdo a la tabla 1 , el 65.8\% de los puntajes fueron iguales o mayores que 15. El máximo puntaje posible era 20 y el mínimo 4 .

\section{Perspectiva del mercado y cliente}

Esta variable fue medida a través de cinco ítems, cuyo puntaje total alcanzado se muestra en la tabla 2:

\begin{tabular}{|c|c|c|c|c|}
\hline \multicolumn{5}{|c|}{ Tabla 2. Perspectiva mercado y clientes } \\
\hline \multirow{4}{*}{} & Frecuencia & Porcentaje & Porcentaje acumulado \\
\hline \multirow{5}{*}{ Válidos } & 13 & 1 & 1.3 & 1.3 \\
\cline { 2 - 5 } & 14 & 5 & 6.6 & 7.9 \\
\cline { 2 - 5 } & 15 & 4 & 5.3 & 13.2 \\
\cline { 2 - 5 } & 16 & 9 & 11.8 & 25.0 \\
\cline { 2 - 5 } & 17 & 8 & 10.5 & 35.5 \\
\cline { 2 - 5 } & 18 & 11 & 14.5 & 50.0 \\
\cline { 2 - 5 } & 19 & 6 & 7.9 & 57.9 \\
\cline { 2 - 5 } & 20 & 15 & 19.7 & 77.6 \\
\cline { 2 - 5 } & 21 & 9 & 11.8 & 89.5 \\
\cline { 2 - 5 } & 22 & 6 & 7.9 & 97.4 \\
\cline { 2 - 5 } & 23 & 2 & 2.6 & 100.0 \\
\cline { 2 - 5 } & Total & 76 & 100.0 & \\
\hline
\end{tabular}

De acuerdo a la tabla 2, el 64.5\% de los puntajes fueron iguales o mayores que 18 . El máximo puntaje posible era 25 y el mínimo 5 .

\section{Perspectiva del entorno interno}

Para esta variable se formularon 6 items y el puntaje total obtenido se muestra en la tabla 3 :

\begin{tabular}{|c|c|c|c|c|}
\hline \multicolumn{5}{|c|}{ Tabla 3: Perspectiva entorno interno } \\
\hline \multicolumn{1}{|c|}{} & Frecuencia & Porcentaje & Porcentaje acumulado \\
\hline \multirow{5}{*}{} & 15 & 1 & 1.3 & 1.3 \\
\cline { 2 - 5 } & 17 & 1 & 1.3 & 2.6 \\
\cline { 2 - 5 } & 18 & 6 & 7.9 & 10.5 \\
\cline { 2 - 5 } & 19 & 3 & 3.9 & 14.5 \\
\cline { 2 - 5 } & 20 & 9 & 11.8 & 26.3 \\
\cline { 2 - 5 } & 21 & 6 & 7.9 & 34.2 \\
\cline { 2 - 5 } & 22 & 11 & 14.5 & 48.7 \\
\cline { 2 - 5 } & 23 & 4 & 5.3 & 53.9 \\
\cline { 2 - 5 } & 24 & 11 & 14.5 & 68.4 \\
\cline { 2 - 5 } & 25 & 10 & 13.2 & 81.6 \\
\cline { 2 - 5 } & 26 & 10 & 13.2 & 94.7 \\
\cline { 2 - 5 } & 27 & 4 & 5.3 & 100.0 \\
\cline { 2 - 5 } & Total & 76 & 100.0 & \\
\hline
\end{tabular}

De acuerdo a la tabla 3, el 65.8\% de los puntajes fueron iguales o mayores que 22 . El máximo puntaje posible era 30 y el mínimo 6 .

\section{Mejoramiento continuo}

Para esta variable se formularon seis ítems y el puntaje total obtenido se muestra en la tabla 4: 


\begin{tabular}{|c|c|c|c|c|}
\hline \multicolumn{5}{|c|}{ Tabla 4: Mejoramiento Continuo } \\
\hline & & Frecuencia & Porcentaje & Porcentaje acumulado \\
\hline \multirow{16}{*}{ Válidos } & 14 & 1 & 1.3 & 1.3 \\
\hline & 15 & 1 & 1.3 & 2.6 \\
\hline & 16 & 3 & 3.9 & 6.6 \\
\hline & 17 & 2 & 2.6 & 9.2 \\
\hline & 18 & 4 & 5.3 & 14.5 \\
\hline & 19 & 5 & 6.6 & 21.1 \\
\hline & 20 & 4 & 5.3 & 26.3 \\
\hline & 21 & 7 & 9.2 & 35.5 \\
\hline & 22 & 5 & 6.6 & 42.1 \\
\hline & 23 & 7 & 9.2 & 51.3 \\
\hline & 24 & 8 & 10.5 & 61.8 \\
\hline & 25 & 12 & 15.8 & 77.6 \\
\hline & 26 & 9 & 11.8 & 89.5 \\
\hline & 27 & 5 & 6.6 & 96.1 \\
\hline & 28 & 3 & 3.9 & 100.0 \\
\hline & Total & 76 & 100.0 & 100.0 \\
\hline
\end{tabular}

De acuerdo a la tabla 4 , el $64.5 \%$ de los puntajes fueron iguales o mayores que 22 . El máximo puntaje posible era 30 y el mínimo 6 .

\section{Eficiencia de las estrategias}

Esta variable fue medida a través de una escala de valoración del 1 a 10 , en donde los extremos eran: 1 (totalmente en desacuerdo) y 10 (totalmente de acuerdo). Los resultados se muestran en la tabla 5 :

\begin{tabular}{|c|c|c|c|c|}
\hline \multicolumn{5}{|c|}{ Tabla 5: Eficiencia de las estrategias } \\
\hline \multicolumn{1}{|c|}{} & Frecuencia & Porcentaje & Porcentaje acumulado \\
\hline \multirow{5}{*}{ Válidos } & 3 & 2 & 2.6 & 2.6 \\
\cline { 2 - 6 } & 4 & 7 & 9.2 & 11.8 \\
\cline { 2 - 6 } & 5 & 13 & 17.1 & 28.9 \\
\cline { 2 - 6 } & 6 & 19 & 25.0 & 53.9 \\
\cline { 2 - 6 } & 8 & 11 & 14.5 & 78.9 \\
\cline { 2 - 6 } & 9 & 3 & 3.9 & 93.4 \\
\cline { 2 - 6 } & 10 & 2 & 2.6 & 97.4 \\
\cline { 2 - 6 } & Total & 76 & 100.0 & 100.0 \\
\hline
\end{tabular}

De acuerdo a la tabla 5, el 71.1\% de los puntajes fueron iguales o mayores que 6. El máximo puntaje posible era 10 y el mínimo 1 .

\section{Análisis bivariado}

En la tabla 6 se ilustran las correlaciones entre la variable eficiencia de las estrategias y perspectiva financiera, perspectiva mercado y clientes, perspectivas del entorno interno y mejoramiento continuo.

\begin{tabular}{|c|c|c|c|c|c|c|}
\hline \multicolumn{7}{|c|}{ Tabla 6. Matriz de correlaciones } \\
\hline & & $\begin{array}{c}\text { Eficiencia de las } \\
\text { estrategias }\end{array}$ & $\begin{array}{l}\text { Perspectiva } \\
\text { financiera }\end{array}$ & $\begin{array}{c}\text { Perspectiva Mercado } \\
\text { y Clientes }\end{array}$ & $\begin{array}{l}\text { Perspectiva En- } \\
\text { torno Interno }\end{array}$ & $\begin{array}{c}\text { Mejoramiento } \\
\text { Continuo }\end{array}$ \\
\hline \multirow{2}{*}{$\begin{array}{c}\text { Eficiencia de las } \\
\text { estrategias }\end{array}$} & Correlación de Pearson & 1 & .187 &, $452^{* *}$ &, $819^{* *}$ &, $351^{* *}$ \\
\hline & Sig. (bilateral) & & .106 & .000 & .000 & .002 \\
\hline \multirow{2}{*}{$\begin{array}{l}\text { Perspectiva finan- } \\
\text { ciera }\end{array}$} & Correlación de Pearson & .187 & 1 &, $328^{\star *}$ &, $401^{* *}$ &, $369^{* *}$ \\
\hline & Sig. (bilateral) & .106 & & .004 & .000 & .001 \\
\hline \multirow{2}{*}{$\begin{array}{c}\text { Perspectiva mercado } \\
\text { y clientes }\end{array}$} & Correlación de Pearson &, $452^{* *}$ &, $328^{* *}$ & 1 &, $460^{* *}$ &, $507^{* \star}$ \\
\hline & Sig. (bilateral) & .000 & .004 & & .000 & .000 \\
\hline \multirow{2}{*}{$\begin{array}{l}\text { Perspectiva entorno } \\
\text { interno }\end{array}$} & Correlación de Pearson &, $819^{* *}$ &, $401^{* *}$ &, $460^{* *}$ & 1 &, $440^{* *}$ \\
\hline & Sig. (bilateral) & .000 & .000 & .000 & & .000 \\
\hline \multirow{2}{*}{$\begin{array}{l}\text { Mejoramiento con- } \\
\text { tinuo }\end{array}$} & Correlación de Pearson &, $351^{* *}$ &, $369^{* *}$ &, $507^{\star *}$ &, $440^{* *}$ & 1 \\
\hline & Sig. (bilateral) & .002 & .001 & .000 & .000 & \\
\hline
\end{tabular}

De la tabla 6 se puede deducir que la eficiencia de las estrategias correlaciona positiva y significativamente con la perspectiva de mercado y clientes, perspectiva del entorno interno y mejoramiento continuo (se observa un valor p casi nulo). Por otro lado, la correlación es fuerte entre eficiencia de las estrategias y perspectiva del entorno interno. 


\section{FORMULACIÓN DEL MODELO MATEMÁTICO}

La hipótesis general de esta investigación establece que la eficiencia de las estrategias es una función de la perspectiva financiera, perspectiva mercado y clientes, perspectiva del entorno interno y mejoramiento continuo. Esto corresponde a un modelo de regresión lineal múltiple, en donde la eficiencia es la variable dependiente y las otras son las variables explicativas o independientes.

Con el soporte del programa informático SPSS v. 22 se realizó el análisis correspondiente, utilizando el algoritmo "hacia atrás" para identificar el mejor modelo.

Los criterios para discriminar el mejor modelo fueron el del coeficiente de determinación, validación por el ANOVA, y significancia de los coeficientes. En la tabla 7 se ilustran los modelos posibles, utilizando el algoritmo "hacia atrás" del programa SPSS.

\begin{tabular}{|c|c|c|c|c|}
\hline \multicolumn{5}{|c|}{ Tabla 7: Resumen del modelo } \\
\hline Modelo & $\mathrm{R}$ & R cuadrado & $\begin{array}{c}\text { R cuadrado } \\
\text { corregida }\end{array}$ & $\begin{array}{c}\text { Error típ. de } \\
\text { la estimación }\end{array}$ \\
\hline 1 &, $842 \mathrm{a}$ &, 708 &, 692 &, 842 \\
\hline 2 &, $841 \mathrm{~b}$ &, 708 &, 696 &, 837 \\
\hline
\end{tabular}

a. Variables predictoras: (constante), mejoramiento continuo, perspectiva financiera, perspectiva entorno interno, perspectiva mercado y clientes.

b. Variables predictoras: (constante), perspectiva financiera, perspectiva entorno interno, perspectiva mercado y clientes.

De acuerdo al criterio del coeficiente de determinación, ambos modelos son válidos (70.8\%). El modelo 1 comprende a las cuatro variables como explicativas, mientras que el modelo 2 descarta la variable mejoramiento continuo.

Por otro lado, todo modelo de regresión tiene que ser validado a través de la significancia de sus coeficientes, a través de la siguiente hipótesis:

Ho: Todos los coeficientes son no significativos.

H1: Por lo menos uno de los coeficientes es significativo.

La verificación se realiza mediante el análisis de varianza (ANOVA), cuyos resultados se muestran en la tabla 8:

\begin{tabular}{|c|c|c|c|c|c|c|}
\hline \multicolumn{7}{|c|}{ Tabla 8: ANOVAa } \\
\hline \multicolumn{2}{|c|}{ Modelo } & $\begin{array}{c}\text { Suma de } \\
\text { cuadrados }\end{array}$ & gl & $\begin{array}{c}\text { Media } \\
\text { cuadrática }\end{array}$ & F & Sig. \\
\hline \multirow{2}{*}{1} & Regresión & 122,389 & 4 & 30,597 & 43,114 &, $000 \mathrm{~b}$ \\
\cline { 2 - 7 } & Residual & 50,387 & 71 &, 710 & & \\
\cline { 2 - 7 } & Total & 172,776 & 75 & & & \\
\hline
\end{tabular}

\begin{tabular}{|c|c|c|c|c|c|c|}
\hline \multirow{4}{*}{2} & Regresión & 122,346 & 3 & 40,782 & 58,225 &, $000 \mathrm{c}$ \\
\cline { 2 - 7 } & Residual & 50,430 & 72 &, 700 & & \\
\cline { 2 - 7 } & Total & 172,776 & 75 & & & \\
\hline
\end{tabular}

De acuerdo a la tabla 8 , el valor $\mathrm{p}$ igual a 0,000 para ambos modelos, indica que los dos son válidos.

Finalmente, la tabla 9 presenta los coeficientes de ambos modelos:

\begin{tabular}{|c|c|c|c|c|c|c|}
\hline \multicolumn{7}{|c|}{ Tabla 9. Coeficientesa } \\
\hline & \multirow{2}{*}{$\begin{array}{c}\text { Modelo } \\
\text { B }\end{array}$} & \multicolumn{2}{|c|}{$\begin{array}{l}\text { Coeficientes no } \\
\text { estandarizados }\end{array}$} & \multirow[t]{2}{*}{$\begin{array}{c}\text { Coeficientes } \\
\text { tipificados }\end{array}$} & \multirow{2}{*}{$t$} & \multirow{2}{*}{ Sig. } \\
\hline & & Error típ. & Beta & & & \\
\hline \multirow{5}{*}{1} & (Constante) & -3.193 & .948 & & -3.366 & .001 \\
\hline & $\begin{array}{l}\text { Perspectiva } \\
\text { financiera }\end{array}$ & -1.128 & .049 & -.188 & -2.597 & .011 \\
\hline & $\begin{array}{c}\text { perspectiva } \\
\text { mercado y } \\
\text { clientes }\end{array}$ & .084 & .048 & .137 & 1.746 & .085 \\
\hline & $\begin{array}{l}\text { Perspectiva } \\
\text { entorno interno }\end{array}$ & .448 & .041 & .840 & 10.857 & .000 \\
\hline & $\begin{array}{l}\text { Mejoramiento } \\
\text { continuo }\end{array}$ & -.008 & .034 & -.019 & -.246 & .807 \\
\hline \multirow{4}{*}{2} & (Constante) & -3.225 & .933 & & -3.456 & .001 \\
\hline & $\begin{array}{l}\text { Perspectiva } \\
\text { financiera }\end{array}$ & -.130 & .048 & -.191 & -2.705 & .009 \\
\hline & $\begin{array}{c}\text { Perspectiva } \\
\text { mercado y } \\
\text { clientes }\end{array}$ & .080 & .045 & .130 & 1.786 & .048 \\
\hline & $\begin{array}{l}\text { Perspectiva } \\
\text { entorno interno }\end{array}$ & .446 & .040 & .836 & 11.129 & .000 \\
\hline \multicolumn{7}{|c|}{ a. Variable dependiente: Eficiencia de las estrategias } \\
\hline
\end{tabular}

En la tabla 9 se observa que para el modelo 2 el valor $\mathrm{p}$, en todos los coeficientes, es menor que $0.05(5 \%)$, lo cual determina que cada uno de los coeficientes sean significativos. En cambio, el modelo 1 presenta dos coeficientes no significativos (valores p mayores que 0,05 ).

Por lo tanto el mejor modelo es el modelo 2, que indica la siguiente relación matemática:

Eficiencia de las estrategias $=-3.225-0.130 \mathrm{PF}$ $+0,080 \mathrm{PMyC}+0,446 \mathrm{PEI}$

En donde:

- PF: perspectiva financiera

- PMyC: perspectiva mercado y clientes

- PEI: perspectiva del entorno interno.

El modelo indica que la variable que explica mejor la eficiencia de las estrategias es la perspectiva del entorno interno (un mayor coeficiente) 


\section{CONCLUSIONES}

1. La eficiencia de las estrategias empresariales depende de las perspectivas financieras, del mercado y clientes en las cuales están inscritas en el entorno interno comprometido, así como del mejoramiento continuo de sus servidores y proveedores, tal como se ha comprobado en el presente estudio.

2. Para el logro de un enfoque sistémico de las estrategias empresariales, éstas se deben tratar en los niveles de las perspectivas señaladas, evaluando y aplicando el marco teórico de la administración de los riesgos, obteniendo mapas de riesgos; así mismo, se requiere para su restructuración apropiadas herramientas como análisis de la industria de Porter, el método Delta, y otras metodologías que permitan comprender los factores críticos de éxito y desarrollar competencias como tomar en cuenta las incidencias de competidores que afecten el desempeño de la empresa, empleando herramientas como la teoría de juegos y la planeación por escenarios.

3. Finalmente, en un adecuado proceso de toma de decisiones y control de su ejecución, se deben tomar consideraciones que formen parte de las estrategias; la existencia de herramientas que son muy amplias y sofisticadas como identificación de riesgos, análisis cuantitativo y semicuantitativo, flujo de caja, análisis de decisiones, arboles de decisiones, simulación de
Montecarlo y otros. Apreciaciones que deben ser tomadas en cuenta durante las prácticas de evaluación. Se complementa con el análisis PESTAL (Político, Económico, Sociocultural, Tecnológico, Ambiental), y el Diamante de Porter. Permiten la construcción de la matriz FODA (Fortaleza, Oportunidades, Debilidades, Amenazas), constituyendo practicas fundamentales en la retroalimentación de la planeación estratégica y logro de su aplicación eficiente.

\section{REFERENCIAS BIBLIOGRÁFICAS}

Bunge, Mario. (1980). Epistemología. Argentina: Editorial XXI.

Cronbach, L. (1951). Coeffient Alpha and the Internal Structure of Test. En: Psycometrika. Septiembre 1951, pp. 297-334.

Díaz, D. 2003. Validación de una escala de medida para la determinación de la calidad de servicio en una institución superior. Centro de competitividad. Documento de trabajo. Recuperado de: <htpp://www.calidad.org/ public/backolds/0993696345digene.htm > .

Porter, Michael (1991). La ventaja Competitiva de las Naciones. Buenos Aires: Editorial Vergara.

Torres, Oscar (1999). "Estudio estadístico del empleo de índices bursátiles como cartera aplicada en condiciones de equilibrio en México", Editado por la Universidad Autónoma Metropolitana de México, México D.F. 